\title{
Molecular Homogeneity in Erbium-Doped Sol-Gel Waveguide Amplifiers
}

\author{
Athanasios Laliotis, Eric M. Yeatman, Munir M. Ahmad, and Weibin Huang
}

\begin{abstract}
High net gain levels have recently been reported in silica-on-silicon waveguide amplifiers, using the sol-gel method of glass deposition. Spectroscopic measurements indicate that high inversion levels are achieved at high erbium concentrations, suggesting a high uniformity in the erbium distribution. Here we show that high inversion and low scattering losses are only achieved in sol-gel waveguides which have had restricted heat treatment and that both degrade upon additional annealing. Evolution of scattering loss spectra are presented, and these are included in a model of the ytterbium-sensitized waveguide amplifiers to show that the gain reduction with heat treatment is not wholly due to pump and signal excess losses, but also to changes in the spectroscopic properties of the erbium ions. In particular, the formation of clusters in which inversion is rapidly quenched is indicated. Thus, we show that the superior performance of the sol-gel method for rare-earth doping of silica waveguides, for amplifier and laser applications, results from its ability to form homogenous molecular structures which are meta-stable and which consequently cannot be formed in glasses that require high-temperature consolidation or annealing.
\end{abstract}

Index Terms-Erbium, integrated optics, optical waveguide components, sol-gel, waveguide amplifiers.

\section{INTRODUCTION}

$\mathbf{E}$ RBIUM-DOPED fiber amplifiers (EDFAs) have achieved very wide deployment in optical communication systems. However, their size and cost continue to restrict their adoption in some important applications. Size is a particular concern, for example, where large numbers of amplifiers would be useful in a confined space, such as around switches in network nodes. Integrated optic waveguides in erbium-doped glass films or monoliths can replace the fiber gain block of an EDFA, leading to erbium-doped waveguide amplifiers (EDWAs). This approach can give a significant size advantage if both small waveguide bend radii, and a higher erbium doping level, can be achieved than what is possible with silica fiber. Cost advantages may also be attained, particularly if the EDWA format is used to reach higher levels of integration and thus to reduce packaging and assembly costs and part count.

The sol-gel method of glass deposition provides a route to high-performance EDWAs in silica-on-silicon. This paper presents evidence that this is due to the creation of a meta-stable molecular structure, containing erbium ions in high concentration, and that this structure deteriorates with further heat

Manuscript received April 16, 2003; revised February 9, 2004. This work was supported by the Engineering and Physical Sciences Research Council and Nortel Networks.

The authors are with the Optical and Semiconductor Devices Group, Department of Electronic and Electrical Engineering, Imperial College London, London SW7 2AZ, U.K.

Digital Object Identifier 10.1109/JQE.2004.828272 treatment. Furthermore, the results indicate that such a meta-stable structure can be achieved while still excluding porosity, $\mathrm{OH}$, and organic groups to a sufficient degree to obtain high amplifier performance and stability. This evidence is supported by fluorescence decay measurements, as well as by amplifier modeling.

\section{FORMATS FOR EDWAS}

The potential for EDWAs has long been recognized, and devices have been demonstrated using a variety of materials systems and fabrication techniques. EDWAs based on ion exchange waveguides in erbium-doped phosphate glass monoliths are perhaps the most advanced [1]. Glass waveguides on silicon substrates, however, offer important advantages in terms of integration possibilities compared to ion exchange guides. The silica-on-silicon system in particular is well established for passive devices such as arrayed waveguide gratings and splitters and provides large wafer sizes, good mechanical properties, well-developed processes, and excellent physical and chemical robustness. Furthermore, the silicon optical bench provides v-grooves for passive single-mode fiber alignment, as well as alignment features and good heatsink properties for optoelectronic die attach.

Silicate glasses have the major disadvantage, however, that the solubility of rare earths in silica is low [2]. Thus, although erbium doped silica waveguide amplifiers have been reported, these have required relatively long waveguides [3]. Consequently, most reported EDWAs have employed other glass types, for example using deposited phosphate [4] and soda-lime [5] waveguides. There are, however, drawbacks to these glass types as well, such as low chemical and thermal stability, lack of suitability of refractive indices, or the need for deposition techniques, such as physical vapor deposition, with their own disadvantages, such as low deposition rate. Therefore, a system that gives high gain levels per unit length, in silica-based waveguides on silicon, remains a very attractive prospect for EDWA implementation.

For photonic devices based on silicate glasses on silicon, two deposition methods have been dominant: flame hydrolysis deposition (FHD), and plasma-enhanced chemical vapor deposition (PECVD). EDWAs have been reported based on both FHD [3] and PECVD [6]. For the former, high levels of phosphorous co-doping were required, and $\mathrm{Er}^{3+}$ concentrations were limited to under $0.5 \mathrm{wt} . \%$ [7]. It is well known that high erbium concentrations tend to reduce the lifetime of the metastable ${ }^{4} \mathrm{I}_{13 / 2}$ energy level, and thus the attainable gain for a given pumping level [8]. When $\mathrm{Er}^{3+}$ ions are sufficiently close to interact (say 
within $\approx 1.5-2 \mathrm{~nm}$ ), cooperative upconversion may take place in which one ion decays to the ground state by transferring its energy to the other, raising it to the ${ }^{4} \mathrm{I}_{9 / 2}$ excited state. While the ion proximities inevitably increase with concentration, the effect can be much more severe if erbium-rich phases separate from the host glass. Such erbium-rich regions are generally referred to as clusters. Most efforts to overcome this difficulty have focused on adding additional dopants to encourage the $\mathrm{Er}^{3+}$ ions to adopt, and retain, well-distributed sites. The sol-gel process, however, offers an alternative approach.

The sol-gel technique has been extensively studied as an alternative glass deposition method for photonics [9]. Typically, in this method, metallorganic precursors such as tetra-ethyl-orthosilicate (TEOS) in an alcohol solution are mixed with catalyzed water and undergo hydrolysis and condensation reactions. A viscous suspension of oxide particles on a nanometer scale is formed which can be spun unto a substrate, whereupon the solvent evaporates rapidly. This increases the particle concentration, and thus the reaction rates, causing the sol to cross-link into a stiff "gel." Further heat treatment is then needed to drive off remaining solvent, to remove residual organic groups, and to collapse the pores. A high degree of shrinkage is involved, and the resulting stress usually limits maximum film thickness; however, cracking can be prevented by an iterative process in which a number of thin layers are deposited and annealed [10]. Based on this approach, buried channel waveguides have been developed with low loss, and passive components such as splitters have been demonstrated [11].

Sol-gel is attractive for active waveguide fabrication partially because of the ease with which dopants can be added. Metalalkoxides are commercially available for a wide variety of metal species, including erbium and other rare earths as well as desirable co-dopants such as aluminum, and although these compounds may have widely differing solubilities and reaction rates, a process can often be developed by which they may be combined in a homogeneous sol. Dopants can also often be added as inorganic salts. In principle, the molecular structure formed in the gel may be quite different from that obtained in melt glasses, since the chemical reactions occur at low temperature, and diffusion within the solid phase is very limited. Furthermore, although the solid thus formed requires heat treatment to remove pores, these pores are on a nanometer scale and so can be collapsed without allowing significant restructuring of the glass by diffusion. Consequently, a fundamental possibility arises: that sol-gel may allow the fabrication of a stable inorganic glass, homogeneous at a molecular scale, which is inaccessible by other techniques. High-concentration doping of Er in silica is then a possible application.

Rare-earth doping of sol-gel monoliths and films has been studied by a number of groups, e.g., [12]-[14]. Relative gain in sol-gel based Er-doped waveguides was first achieved by the use of a strip loaded format [15], but high coupling and background propagation losses prevented net fiber-to-fiber gain from being reached. Relative gain was also achieved in Er-doped hybrid inorganic-organic sol-gel waveguides, by minimizing $\mathrm{OH}$ content [16]. More recently, we have fabricated erbium-doped buried channel waveguide devices. These buried channel sol-gel guides showed low background loss levels of about $0.1 \mathrm{~dB} / \mathrm{cm}$, with high $\mathrm{Er}_{2} \mathrm{O}_{3}$ doping levels of $0.25 \mathrm{~mol} \%$. This system was the first to achieve net fiber-fiber gain in a sol-gel based Er-doped waveguide amplifier, this net gain being $5.75 \mathrm{~dB}$ in a $5-\mathrm{cm}$-long waveguide [17]. Per unit length, this we believe is the highest reported in the silica-on-silicon format.

It has generally been assumed that the ability of Er-doped sol-gel films to sustain high inversion at high doping levels results from a lower level of erbium clustering and that this in turn results from the formation of a uniform molecular structure in the gel which is retained in the final glass. However, previous studies have not clearly demonstrated this cause. Direct physical measurement of the clusters in thin films is very challenging. One method that has been investigated is extended X-ray absorption fine structure (EXAFS) [18], allowing the neighboring atoms to Er ions to be determined. The first coordination shell was found to consist of 6-7 oxygen atoms, and Er ions were also not detected in the second nearest neighbor (SNN) positions, even for the highest Er concentrations (1.75 mol\%). This suggests that at least pure erbium oxide phases were not formed. However, the accuracy of EXAFS for SNNs is limited, and little information can be obtained for more distant neighbors, although cooperative relaxation effects can be strong at ranges of several atomic distances. In this study, we have evaluated the uniformity of erbium distribution using fluorescence measurements and thus demonstrate that a uniform structure is achieved which is meta-stable by showing that further heat treatment induces formation of erbium rich regions.

\section{DeVICE PROCESSING}

Devices were fabricated as in [17], with only the active guiding layer made by sol-gel. The buffer layer was formed by steam oxidation of the silicon substrate, over which the Er-doped layer was deposited. This layer was alumino-phosphosilicate glass doped with erbium and ytterbium; the phosphorous reduces the process temperatures and helps exclude residual $\mathrm{OH}$ groups (which quench the gain), while ytterbium increases pump absorption and transfers energy to the erbium. The silicon precursor was TEOS. Both of the rare earths, and the aluminum, were added to the sol as salts. The active layer composition was in the molar ratio

$$
\mathrm{SiO}_{2}: \mathrm{P}_{2} \mathrm{O}_{5}: \mathrm{Al}_{2} \mathrm{O}_{3}: \mathrm{Er}_{2} \mathrm{O}_{3}: \mathrm{Yb}_{2} \mathrm{O}_{3}=87: 10: 2.5: 0.25: 0.25
$$

which corresponds to $\mathrm{Er}$ and $\mathrm{Yb}$ concentrations of $1.2 \mathrm{wt} . \%$ (or $\approx 10^{20}$ ions $/ \mathrm{cm}^{3}$ ) each. The sol was catalyzed with $\mathrm{HCl}$, and the water-to-TEOS ratio $R$ was 2 .

The sol-gel active layer was consolidated after deposition for $1 \mathrm{~h}$ at $1000{ }^{\circ} \mathrm{C}$ in air, and was approximately $4.5 \mu \mathrm{m}$ thick after this step. This consolidation step is typically carried out for sol-gel as well as other deposition techniques, in order to remove residual porosity. Such porosity can lead to instability of the optical and physical properties by allowing the ingress of water and other contaminants, increasing scattering losses in the waveguide, and increasing sidewall roughness of the etched guiding ridges. The choice of temperature and time for this annealing step is a compromise between the need to remove the porosity and the need to avoid crystallization or phase separation. In [19], extensive measurements of the structure of sol-gel silica films, 


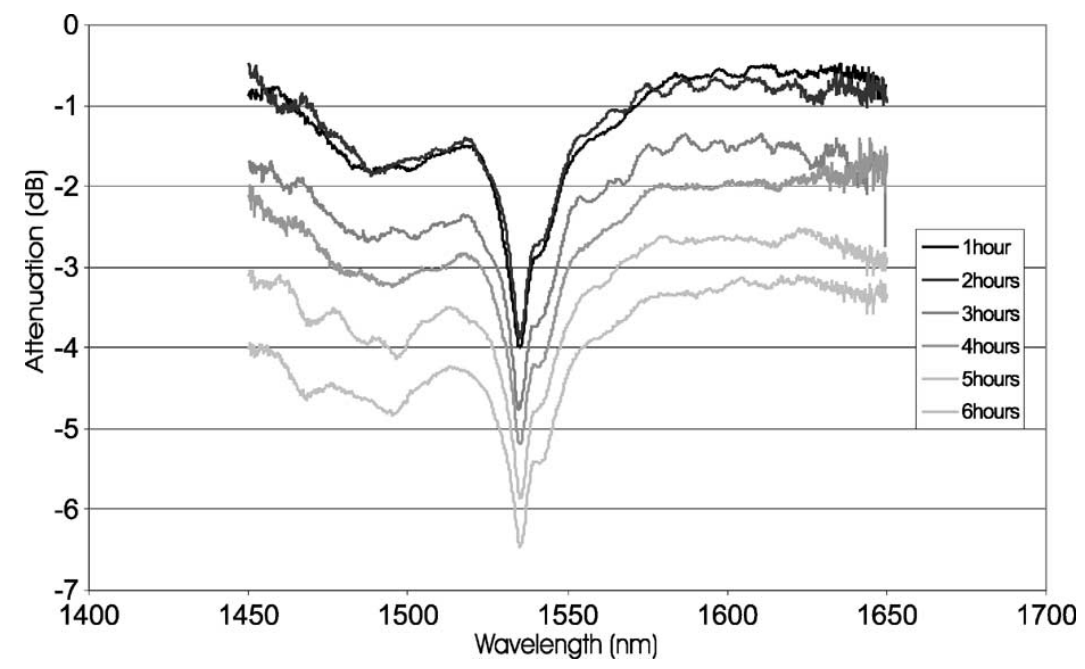

Fig. 1. Absorbtion spectra of sol-gel EDWA for six consecutive annealing stages.

as a function of annealing temperature and synthesis parameters, were reported. It was found that annealing at $800^{\circ} \mathrm{C}$ for 30 min was sufficient to remove porosity accessible to water, but $1000^{\circ} \mathrm{C}$ was required to stabilize refractive index; both of these conclusions are independent of the molar ratio of water to silicon in the sol. Therefore, we chose the latter temperature, with the time extended to $1 \mathrm{~h}$ to allow for any slowing of consolidation rate by the dopants. Following consolidation, grazing angle $\mathrm{X}$-ray diffraction was carried out to determine whether any crystallization had taken place; no measurable peaks were observed.

Straight waveguide ridges of widths from 3 to $6 \mu \mathrm{m}$ were then formed by reactive ion etching through the complete active layer thickness. In contrast to previous passive buried channel devices, reflow of these ridges was not performed. A cladding layer was then formed in boro-phosphosilicate glass deposited by PECVD. The index difference was about $1 \%$. Finally, individual dies were cleaved from the wafer for testing.

Both aluminum and phosphorous are known to improve the homogeneous dispersion of rare earth ions in silicate glasses. Although the mechanisms are not well understood, a solvation shell model is frequently cited [20]. Aluminum co-doping has been the most studied, and recent thermodynamical analysis [21] has suggested that there is a critical value of aluminum to rare-earth ion ratio $R_{c}$ required to avoid aggregation, which depends on the rare earth concentration and moreover on the annealing temperature and time exposure. According to these observations, and the consolidation temperatures and erbium concentrations in this case, the required $R_{c}$ should be 8-10, similar to the $\mathrm{Al}$ :Er ratio used. Appropriate ratios when $\mathrm{Yb}$ and phosphorous are included are less clear. In any case, the analysis of [21] suggests that the resulting structure should be stable with further annealing at the given temperature, which is not what we find.

In order to examine the thermodynamic stability of the composition, we further annealed the guides at $1000{ }^{\circ} \mathrm{C}$ in 1-h steps, for five consecutive cycles, after each of which the performance was remeasured. This annealing was conducted in air in a conventional furnace, following removal of coupling oil and other contaminants in solvent reflux. Since the layers are already fully consolidated, and the temperature of this additional annealing is well below the reflow temperature, no dimensional changes were expected. This was confirmed by scanning electron microscopy of waveguide cross-sections, and by coupling loss measurements as described below. We designate $T$ as the total annealing time at $1000{ }^{\circ} \mathrm{C}$, including the initial 1-h consolidation.

\section{SPECTROSCOPIC MEASUREMENTS}

Optical characterization measurements were repeated for core widths $w$ ffof 3, 4, 5, and $6 \mu \mathrm{m}$. All the amplifiers had an active layer height of about $4.5 \mu \mathrm{m}$ and were $2 \mathrm{~cm}$ long. The measurements consisted of insertion loss, absorption and emission spectra, and fluorescence lifetime. All four waveguide widths displayed similar behavior.

\section{A. Insertion Loss Measurements}

The absorption spectra of the amplifiers were measured after each annealing cycle, using an Agilent spectrometer $86142 \mathrm{~B}$ and its internal light-emitting diode source, emitting from 1450 to $1650 \mathrm{~nm}$. The results are shown in Fig. 1 for $w=5 \mu \mathrm{m}$. The spectral shape remains substantially unchanged as annealing progresses, as does the magnitude of the $\mathrm{Er}$ absorption peak. This suggests that the core composition has remained unchanged, that no significant diffusion of erbium or other ions into the cladding has taken place, and that there is no significant variation of the refractive index of the core. From these measurements we can extract the background propagation losses; we assess these at the 1620-nm wavelength in order to minimize the effects of the erbium $\mathrm{I}_{13 / 2}$ band absorption, while staying close to the signal wavelength. The results are shown in Fig. 2 for two waveguide widths, indicating the consistency of the results obtained.

To establish the fact that the induced heat treatment had not changed the waveguide shape or refractive index, the coupling loss was measured for the $T=6 \mathrm{~h}$ sample. This was done by measuring the absorption spectrum using single-mode fiber (SMF) at device input and output and again using a multimode fiber (MMF) from the output to the spectrometer, assuming 


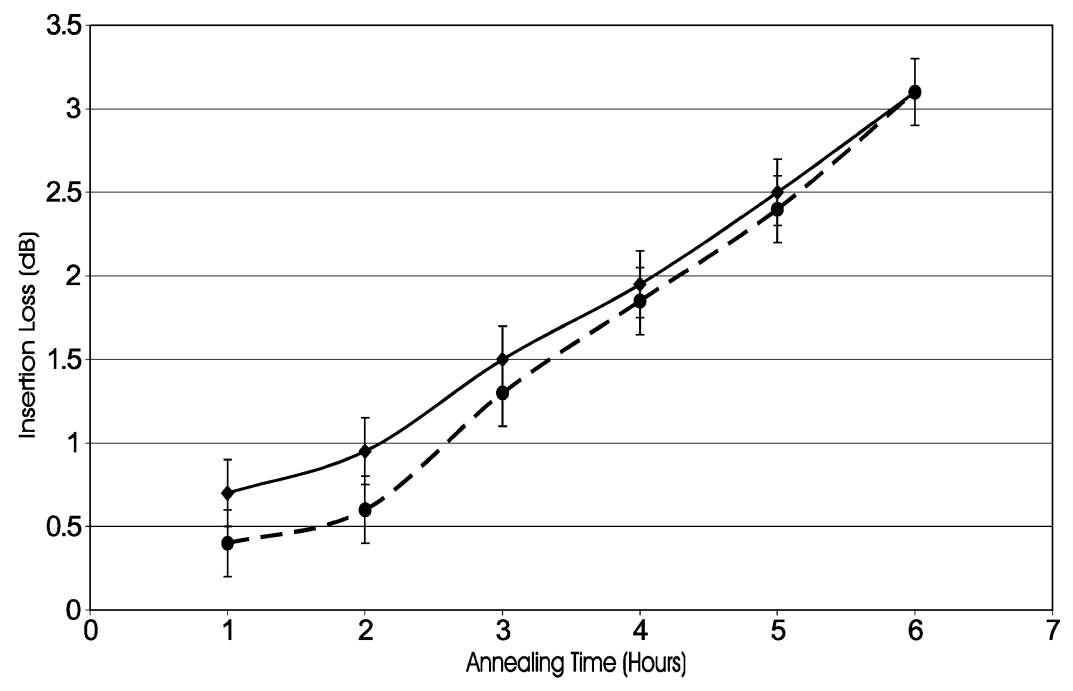

Fig. 2. Amplifier insertion loss at $1620 \mathrm{~nm}$ versus annealing time for waveguide widths of $5 \mu \mathrm{m}$ (solid line) and $4 \mu \mathrm{m}$ (dashed line).

TABLE I

PRopagation LosSES (dB/cm) From MEASUREMENTS AT 1300 AND $1620 \mathrm{~nm}$ SIGNAL WAVELENGTHS AND EXTRAPOLATED AT $1000 \mathrm{~nm}$

\begin{tabular}{l|l|l|l}
\hline \multicolumn{1}{c|}{ Wavelength } & $1000 \mathrm{~nm}$ & $1300 \mathrm{~nm}$ & $1620 \mathrm{~nm}$ \\
\hline 1hour & 0.9 & 1.1 & 0.15 \\
\hline 2hours & 1.95 & 0.35 & 0.275 \\
\hline 3hours & 3.9 & 1.25 & 0.55 \\
\hline 4hours & 5.5 & 1.75 & 0.775 \\
\hline 5hours & 7.5 & 2.40 & 1.05 \\
\hline 6hours & 9.6 & 3 & 1.35 \\
\hline
\end{tabular}

negligible coupling loss from the device to the MMF. The difference gives a single device-SMF coupling loss. The results show that the coupling losses of the amplifier to SMF were of the order of $0.1-0.2 \mathrm{~dB}$ per facet. Similar results were obtained by using the cut back method in [17]. Waveguide propagation losses were measured at 1620 and $1300 \mathrm{~nm}$, well outside the erbium absorption bands, by subtracting coupling loss (determined as above) from overall insertion loss. The results are shown in Table I. The wavelength variation of loss fits well to a Rayleigh model (i.e., proportional to $\lambda^{-4}$ ), so excess loss figures at the pump wavelength were extrapolated accordingly. It is not possible to separate the various factors contributing to excess propagation loss; however, erbium-rich nano-clusters of higher refractive index than the host glass could act as small scattering spheres, and such a mechanism should follow a Rayleigh loss profile.

\section{B. Internal Gain Measurements}

At every annealing step, both the spontaneous emission spectra and the gain spectra of the waveguides were also recorded. For this purpose, we used a pump laser diode of maximum power (into the waveguides) $180 \mathrm{~mW}$ and the signal diode as above in the case of gain. A consistent drop in net gain with annealing time was observed, as illustrated in Fig. 3. To interpret these results more clearly, the internal gain was calculated (i.e., the effect of background losses was subtracted), since the excess losses contribute a substantial drop in the overall spectrum, a phenomenon which needs to be separated from the actual population inversion of the erbium ions. Both of those parameters can be seen as a function of anneal time in Fig. 4. Although the net gain has shown a consistent drop, the internal gain presents a temporary plateau in the third and fourth steps of the process. The interpretation of these results is complicated, partially because the excess losses at the pump wavelength become severe for the longest anneal times. For a better understanding of the experimental results, therefore, we have employed waveguide modeling.

\section{AMPLIFIER MODELING}

Waveguide amplifier modeling has been extensively studied over a number of years. Although EDWAs operate under the same principles as EDFAs, there are a number of problems arising from the high levels of erbium concentration which make it much more difficult to model the behavior of the former. One of the first studies on the subject was done by di Pasquale and Zoboli [22] using the finite element approach for simulating the mode profiles of a waveguide, combined with the solution of the amplifier propagation equations. This first study included only the effects of erbium up-conversion, but was developed over the years to include more complex phenomena, such as ytterbium sensitization and excited state absorption [23]-[26], by many authors. More recently, the method of lines as a beam propagation method was used successfully for this application [27]. The effects of erbium clustering have so far been mainly modeled by the ion-pair method [28]-[32] which assumes that a fraction of the ions act as pairs with an effective lifetime much lower than that for the single ions. This model has also been extended to include the effects of a larger number of ions (e.g., ion triplets).

Creating a suitable model to include the heavily annealed cases requires modeling of the nature of clusters and the associated physical mechanisms that cause the rapid de-excitation 


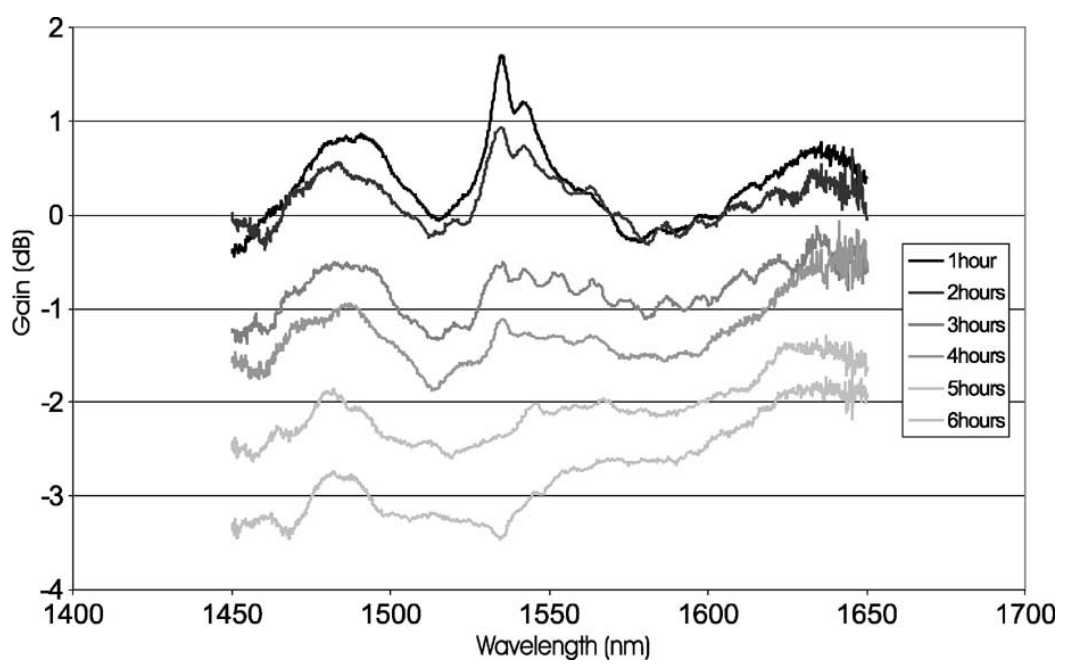

Fig. 3. Emission spectra of sol-gel EDWA for six consecutive annealing stages.

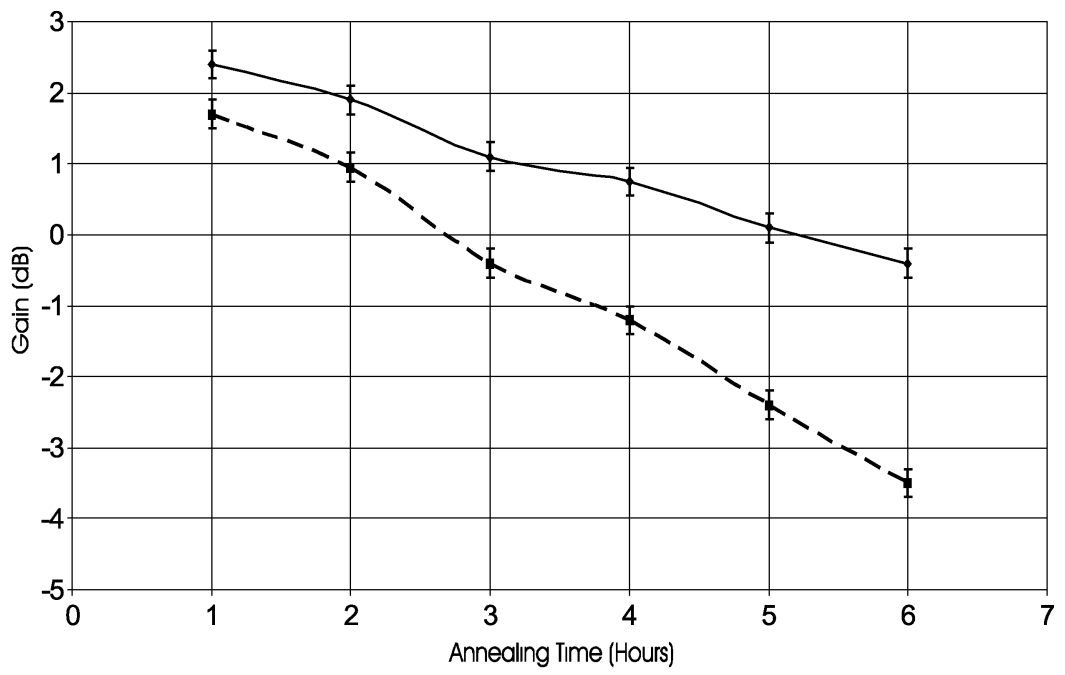

Fig. 4. Amplifier net (dashed line) and internal gain (solid line) versus annealing time at $1535 \mathrm{~nm}$.

observed, and, since little physical information is obtainable, estimates and assumptions must be made. Although the ion pair model is well established and has given good results in some cases [30], in this case the high propagation losses reached suggest that the clusters reach a considerable size. We have therefore chosen to apply a homogeneous model, since this approximation will provide insight into the interpretation of the experimental results.

The rate equations used in the model are given below, with levels $N_{1}, N_{2}, N_{3}$, and $N_{4}$ representing the populations of the erbium ion ground $\left({ }^{4} \mathrm{I}_{15 / 2}\right)$, metastable $\left({ }^{4} \mathrm{I}_{13 / 2}\right)$, and excited $\left({ }^{4} \mathrm{I}_{9 / 2},{ }^{4} \mathrm{~F}_{7 / 2}\right)$ states, respectively, and $N_{5}$ and $N_{6}$ the populations of the ytterbium ion ground $\left({ }^{2} \mathrm{~F}_{5 / 2}\right)$, and excited $\left({ }^{2} \mathrm{~F}_{7 / 2}\right)$ states, respectively, given as follows:

$$
\begin{aligned}
\frac{d N_{1}}{d t}= & W_{12} N_{2}-W_{21} N_{1}+W_{13} N_{3}-W_{31} N_{1} \\
& +\frac{N_{2}}{\tau_{2}}+\frac{N_{3}}{\tau_{3}}+K_{2} N_{2}^{2}+K_{3} N_{3}^{2}-C_{u p} N_{1} N_{6} \\
\frac{d N_{2}}{d t}= & W_{12} N_{1}-W_{21} N_{2}-\frac{N_{2}}{\tau_{21}}+\frac{N_{3}}{\tau_{32}}-2 K_{2} N_{2}^{2}
\end{aligned}
$$

$$
\begin{aligned}
\frac{d N_{3}}{d t}= & W_{13} N_{1}-W_{31} N_{3}-\frac{N_{3}}{\tau_{32}}-\frac{N_{3}}{\tau_{31}} \\
& +\frac{N_{4}}{\tau_{41}}-2 K_{3} N_{3}^{2}-C_{u p} N_{1} N_{6} \\
\frac{d N_{4}}{d t}= & -\frac{N_{4}}{\tau_{41}}+K_{2} N_{2}^{2}+K_{3} N_{3}^{2} \\
\frac{d N_{5}}{d t}= & W_{65} N_{6}-W_{56} N_{5}+\frac{N_{6}}{\tau_{65}}+C_{u p} N_{1} N_{6} \\
\frac{d N_{6}}{d t}= & W_{56} N_{5}-W_{65} N_{6}-\frac{N_{6}}{\tau_{65}}-C_{u p} N_{1} N_{6} .
\end{aligned}
$$

The constants $W_{i j}, \tau_{i j}$ represent the transition rates and lifetimes, respectively, between levels $i$ and $j$, whereas $K_{2}$ and $K_{3}$ are the cooperative up-conversion constants from levels $I_{13 / 2}$ and $I_{9 / 2}$. The ytterbium sensitization is described through levels 5 and 6, and $C_{\text {up }}$ is the erbium-ytterbium interaction constant.

In this case, we used the method of lines to propagate both pump and signal fields. The details of this method are given in [27]. Here we state the basic principles: 1) the lateral direction ( $x$ axis) of the amplifier is discretised in lines of uniform refractive index; 2) the amplifier is excited with both signal and pump fields at its input end; and 3) at each line we solve the above set 

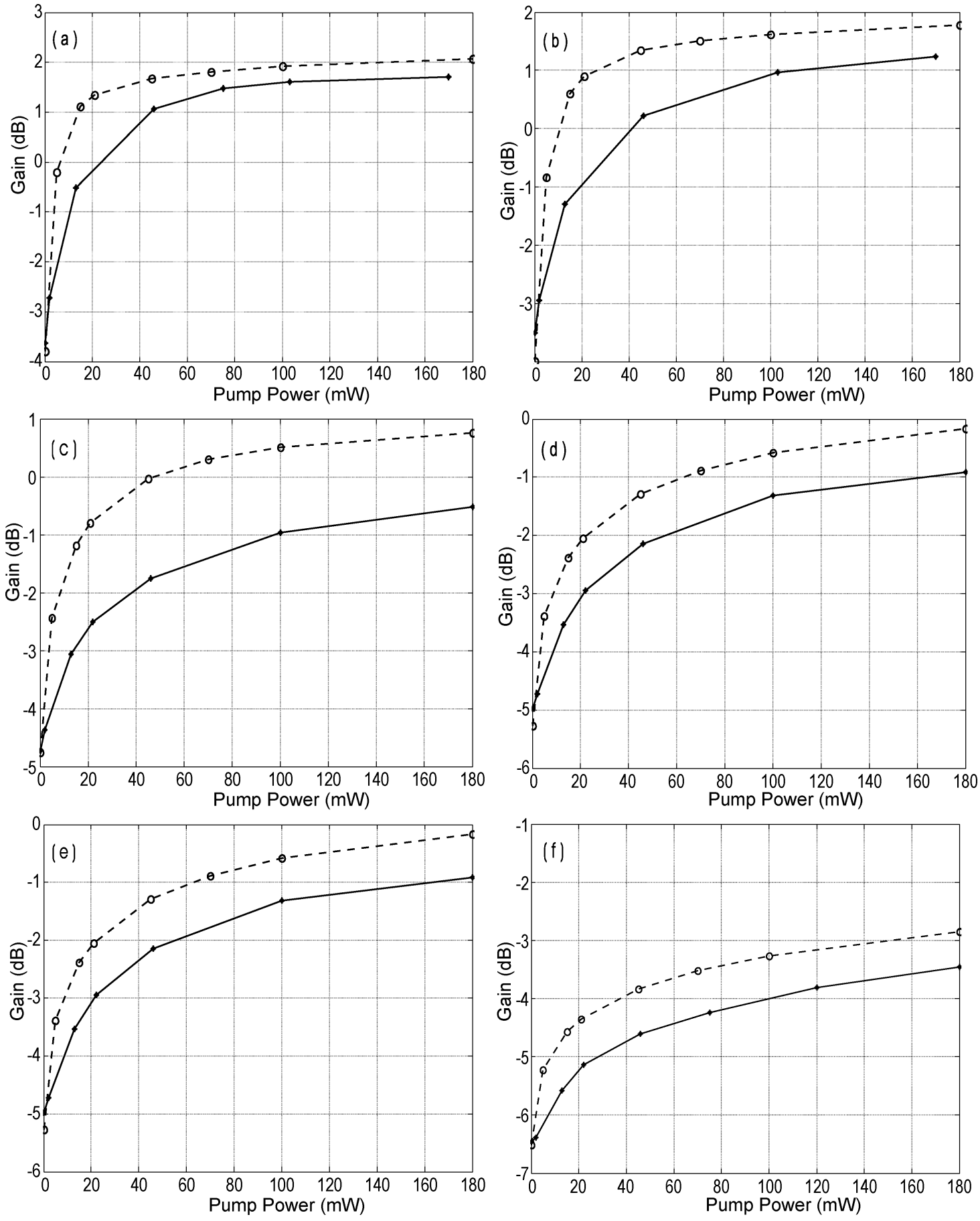

Fig. 5. Gain versus pump power for each annealing step. The dashed curves show the expected homogeneous case degradation, whereas the solid curves show the experimentally measured results. (a)-(f) $T=1-6 \mathrm{~h}$, respectively.

of rate equations and calculate the profile of the complex refractive index through

$$
\begin{aligned}
\varepsilon_{r}=n^{2}(x)-j\left(\frac{\lambda n(x)}{2 \pi}(\right. & N_{2}(x, z)^{*} \sigma_{e}(\lambda) \\
& \left.\left.-N_{1}(x, z)^{*} \sigma_{a}(\lambda)\right)+\alpha_{\text {signal }}\right)
\end{aligned}
$$

where $n$ is the real refractive index of the material, $\sigma_{e}$ and $\sigma_{a}$ are the emission and absorption cross sections, respectively, and $\alpha_{\text {signal }}$ is the background loss coefficient at the signal wavelength. In (2) the $z$ axis corresponds to the direction of propa- gation. Then: 4) we propagate both pump and signal for a short distance and 5) steps 3) and 4) are repeated.

\section{A. Results and Discussion}

The amplifier parameter values used are given in Table II. Most have been measured directly from our samples, but for a few we have relied upon previously reported values. The ytterbium lifetime $t_{6}$ and the $t_{4}, t_{32}$, and $t_{3}$ values are reported in [25] and [26]. For the erbium-erbium ion interactions, experimentally reported [26], [33] values of the cooperative up-conversion constant $K_{2}$ range from $0.5-0.8 \times 10^{-5} \mu \mathrm{m}^{3} / \mathrm{s}$, and the ytter- 
TABLE II

COEFFICIENTS USED FOR THE NUMERICAL MODELING

\begin{tabular}{l|l}
\hline$\lambda_{\mathrm{s}}=1535 \mathrm{~nm}$ & $\mathrm{t}_{31}=2 \times 10^{-5} \mathrm{sec}$ \\
\hline$\lambda_{\mathrm{p}}=980 \mathrm{~nm}$ & $\mathrm{~K}_{2}=\mathrm{K}_{3}=0.5 \times 10^{-5} \mu \mathrm{m}^{3} / \mathrm{sec}$ \\
\hline$\tau_{2}=8 \mathrm{msec}$ & $\mathrm{C}_{\mathrm{up}}=0.5 \times 10^{-4} \mu \mathrm{m}^{3} / \mathrm{sec}$ \\
\hline$\tau_{32}=1 \times 10^{-5} \mathrm{sec}$ & $\sigma_{12}=3.7 \times 10^{-13} \mu \mathrm{m}^{2}$ \\
\hline$\tau_{4}=1 \times 10^{-9} \mathrm{sec}$ & $\sigma_{21}=3.7 \times 10^{-13} \mu \mathrm{m}^{2}$ \\
\hline$\tau_{6}=1 \mathrm{msec}$ & $\sigma_{13}=\sigma_{31}=2.58 \times 10^{-13} \mu \mathrm{m}^{2}$ \\
\hline
\end{tabular}

bium-sensitized luminescence constant follows according to the Dexter theory [26] to be about $C_{\mathrm{up}}=0.5 \times 10^{-4} \mu \mathrm{m}^{3} / \mathrm{s}$. Based on the experimental data reported above, which correspond to a homogeneous case, gain versus pump power was modeled. The result is shown in Fig. 5(a) for the least annealed sample measured. It can be seen that the fit between experimental and modeled results is not very close, suggesting that even in the original $(T=1 \mathrm{~h})$ samples the material structure is not fully homogeneous and thus that some further process optimization is possible. In Fig. 5(b)-(f), the simulated gain versus pump power is compared to measured results for the higher annealing times. For the first steps, the fit becomes worse, i.e., the measured gain drops more rapidly than the modeling predicts. Since the adjustment to the model is to increase the background propagation losses at signal and pump wavelengths, these loss increases are thus shown not to account fully for the change of characteristics with annealing, i.e., that the fluorescence behavior of the rare-earth ions is also altered. Fig. 5 indicates that, at $2-3 \mathrm{~h}$ of heat treatment, the difference between experimental and simulated curves is at a maximum. A possible interpretation is that changes in the erbium ion local environment, e.g., through phase separation, take place primarily until that point, after which the fraction of erbium ions in clustered phases stops increasing. The further decreasing of gain is then due mainly to the increase of the background propagation losses.

In order to test the validity of this interpretation, homogeneous least-square fits of the experimental curves were obtained by varying the $K_{2}$ and $C_{\text {up }}$ parameters. After obtaining a fit for each anneal time, the drop of internal gain that would be expected from further increases in propagation losses was modeled. In Fig. 6, the results are plotted. Beyond the fourth hour of annealing, the curves converge, showing that background loss increases can account well for the alteration in fluorescence properties beyond this point. This supports the proposition that, for the earlier annealing stages, the erbium environment is altering. In Fig. 2, it can also be seen that the increase in propagation losses with annealing time becomes approximately linear after the first three hours.

The values of the erbium up-conversion constant $K_{2}$ which correspond to the best homogeneous fits are plotted in Fig. 7, as a function of annealing time. This fitting, of course, overlooks the fact that this constant would be expected to take different values inside and outside any erbium clustered phase. After the third hour, the value stabilizes, again suggesting an attained stability of erbium local environment at this point.

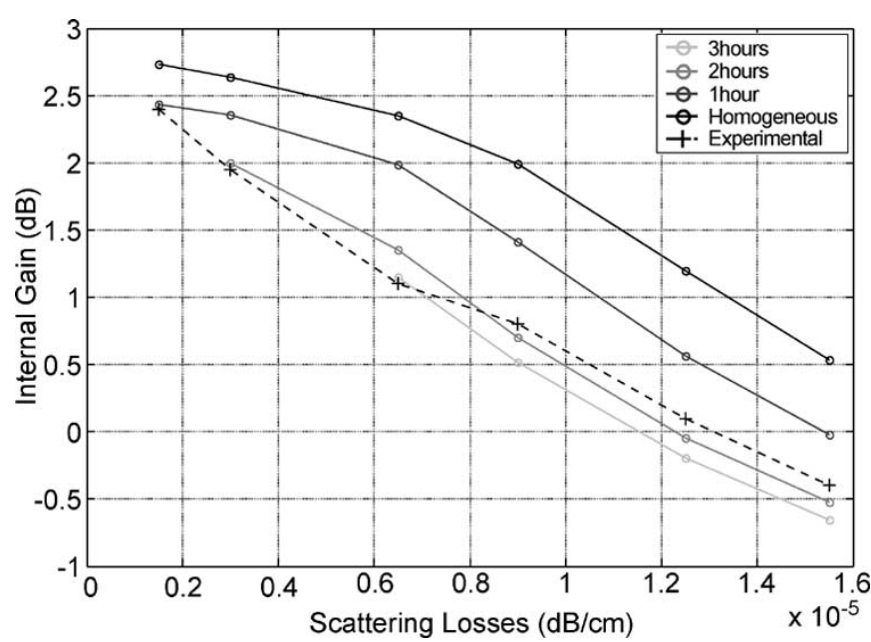

Fig. 6. Modeled dependence of gain on scattering loss at signal wavelength, for $K_{2}$ and $C_{\text {up }}$ from the literature (homogeneous case) and fitted values for $T=1-, 2-$, and 3-h measurements, and measured results (dashed line).

A possible mechanism is now needed to account for the further increase in propagation losses for the final stages of annealing. It has been suggested by various researchers that erbium-rich clusters after extensive heat treatment follow Ostwald ripening dynamics. This means that the clusters tend to reduce in density while increasing in mean size. From [34] and [35], the relation for Ostwald ripening is

$$
d \propto D t^{\frac{1}{3}}
$$

where $d$ is the cluster diameter, $D$ is a diffusion constant dependent on temperature, and $t$ is the annealing time.

The dependence of propagation losses on the size and refractive index of micro-clusters can be modeled as

$$
\operatorname{Loss}(\mathrm{dB} / \mathrm{cm})=\frac{\pi d^{3}}{8 \lambda^{4}}\left[\frac{\left(\frac{n_{2}}{n_{1}}\right)^{2}-1}{\left(\frac{n_{2}}{n_{1}}\right)^{2}+2}\right]^{2} 10 \beta .
$$

In this model, clusters are assumed to be spheres of radius $d$ and of a refractive index $n_{2}$, while the refractive index of the rest of the core is $n_{1}$ and $\beta$ is the fractional volume occupied by the clusters.

If we assume that the clusters have a common composition and structure, as suggested in [21], then $n_{2}$ and $n_{1}$ should remain approximately constant. If we further assume that, after the first three hours of annealing, the fractional cluster volume $\beta$ reaches an equilibrium, then according to (4) the scattering losses of the waveguide will increase in the third power of the cluster diameter. By combining (3) and (4), we then obtain a scattering loss that is linearly increasing with annealing time, which agrees with the results in Fig. 2.

\section{AMPLIFIER FLUORESCENCE DECAY}

It has been suggested [33] that the amplifier fluorescence decay follows an almost quadratic drop in the first few milliseconds $\left(-C^{*} N_{2}^{2}\right)$, followed by a linear drop $\left(-N_{2} / t_{2}\right)$ which is purely dependent on the lifetime of the meta-stable level $\left(t_{2}\right)$. 


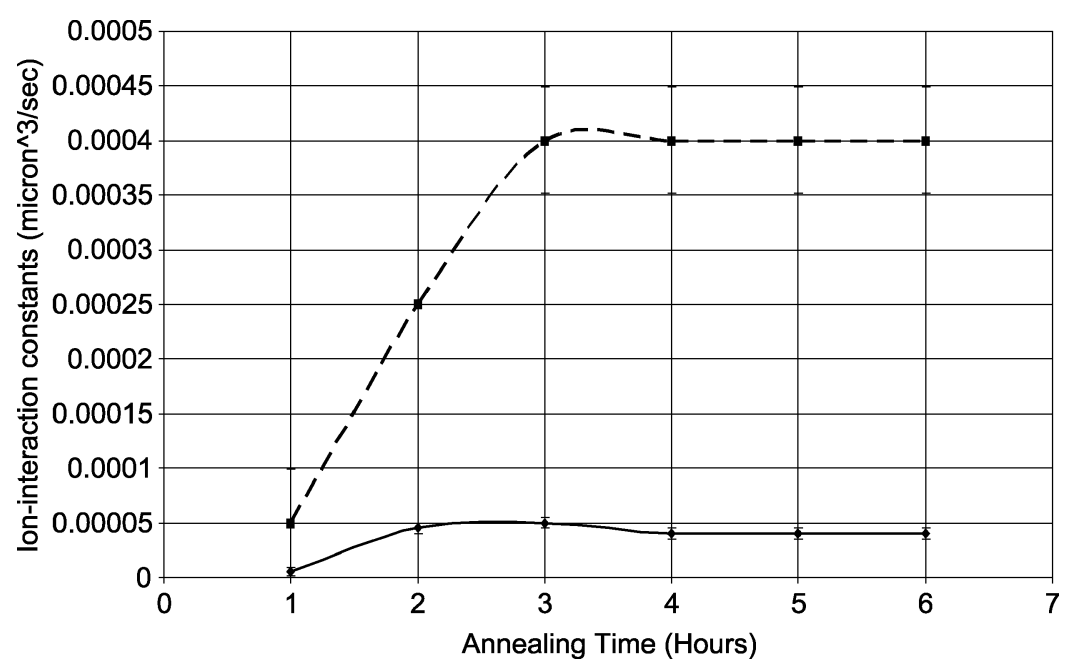

Fig. 7. Ion interaction parameters for best homogeneous fits at each annealing step: erbium-erbium (solid line) and erbium-ytterbium (dashed line).

In our case, there are clear deviations from this behavior, suggesting two different types of erbium population in the amplifier. There is evidence of a very fast decay process, typically attributed to erbium clustering [36]; however, in our case, this phenomenon was evident with levels of pump power as low as $45 \mathrm{~mW}$, in contrast to a few watts usually required for such measurements. A possible explanation is that the presence of ytterbium ions inside the clustered phases can lead to reasonable levels of erbium inversion and thus reduce the detrimental effects of clustering on the amplifier's performance.

Lifetime measurements were performed by mechanically chopping the pump light, after which about $45 \mathrm{~mW}$ could be coupled into the amplifying guides. To increase the degree of inversion at this level, a sample of about $1 \mathrm{~cm}$ in length was measured. Ideally, the decay of erbium fluorescence in the homogeneous case is predicted by solving the rate equations (1) in the nonsteady state. When ytterbium is not present, this complex system could be approximated by

$$
\frac{d N_{2}}{d t}=-\frac{N_{2}}{\tau_{2}}-2 K_{2} N_{2}^{2}
$$

which can be solved analytically [37] and predicts an initial quadratic decay, due to the effects of erbium upconversion, followed by a linear decay, due to the lifetime of the meta-stable level. Unfortunately, for ytterbium co-doping, such an analytic solution cannot be found and one has to rely on numerical solution for the set of differential equations. For a typical amplifier, the results of the two cases differ only in the first half millisecond (the precise time depending on the value of $C_{\mathrm{up}}$ ), where a temporary plateau is observed due to the fact that ytterbium ions quickly transfer their energy through sensitized luminescence to erbium ions, thus keeping the erbium population inversion constant. It has been suggested in [38] that $C_{\text {up }}$ can be measured from that plateau; however, in our case this was not possible.

The fluorescence decay was measured for one and three hours of heat treatment. The results are presented in Fig. 8(a). In addition, the best fits to those curves are shown, again by varying $K_{2}$ and $C_{\text {up }}$ parameters, according to the theoretical model. In the first case, the simulated and experimental results seem to
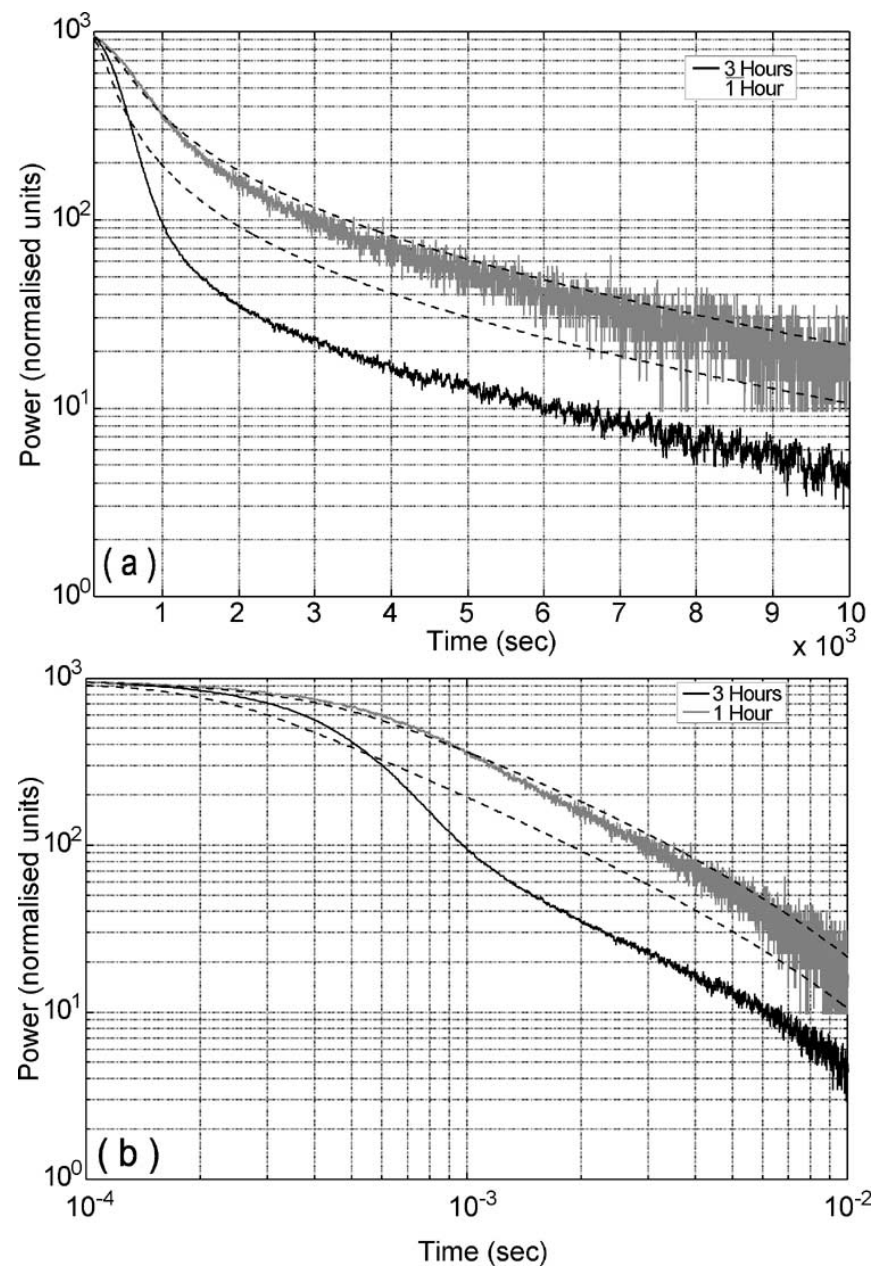

Fig. 8. Fluorescence decay measurements for two different annealing stages. Homogeneous fits are represented by dashed lines. (a) Linear-log plot. (b) Log-log plot.

match relatively well whereas in the second case the mismatch is more obvious. However, when the results are plotted in a logarithmic scale, Fig. 8(b), the differences between homogeneous theory and experimental results is more pronounced, and the deviations of our experimental results from the expected quadratic 
behavior are evident even for restricted heat treatment. Also, the fast decay time constant due to erbium clustering is not as small as has been reported by other researchers. This, and the low levels of pump power, suggests that both rare-earth species have clustered together and thus that the ion pair model often used to describe the physics of clusters is inappropriate in this case. Ideally, a full model including the physical mechanisms for both clustered and unclustered erbium populations is required.

Finally, other quenching mechanisms should be considered besides clustering and homogeneous upconversion. These other mechanisms will be due to impurities, of which the most important are $\mathrm{OH}$ groups, the quenching effects of which have been extensively studied [39]. OH groups can be detected by a broad absorption band in the near infrared, which was not observed in these samples. Furthermore, additional annealing would not be expected to increase the level of $\mathrm{OH}$ impurity (or of other impurities) in a consolidated film. Therefore, $\mathrm{OH}$ can be discounted as a cause of the increased quenching.

\section{CONCLUSION}

Previous work on Er-doped silicate waveguide amplifiers has shown superior performance when sol-gel fabrication is used, indicating that relatively uniform Er distribution is achieved. Our results further indicate that erbium precipitation in sol-gel amplifier samples is induced by high-temperature annealing, increasing with time until it reaches a point of relative stability after the first $2-3 \mathrm{~h}$ at $1000{ }^{\circ} \mathrm{C}$. This is related to a substantial drop in the amplifiers' net gain and an increase in the pump power requirements. Beyond this point, additional heat treatment further increases the scattering losses in a linear fashion, thus further degrading the performance. This behavior can be explained by an increase of cluster size. These results give the first strong indication of the meta-stable nature of sol-gel material and its possible advantages against other methods of fabricating silica-based EDWAs of high rare earth content. In addition, since the reduction in performance is monotonic with additional annealing, these results indicate that amplifier performance can be enhanced further by decreasing to a minimum the consolidation time and temperature, while still eliminating porosity.

Fluorescence decay behavior has also been measured for two points in the annealing process. To our knowledge, this is the first time that the effects of clustering in erbium lifetime measurements have been displayed for the same amplifier.

\section{ACKNOWLEDGMENT}

The authors are grateful to R. R. A. Syms of Imperial College, London, and T. V. Clapp, S. M. Ojha, and J. Barr, formerly of Nortel Networks, for assistance with sample fabrication and helpful discussions.

\section{REFERENCES}

[1] D. Barbier, P. Bruno, C. Cassagnettes, M. Trouillon, R. L. Hyde, A. Kevorkian, and J. M. P. Deleveauxl, "Net gain of $27 \mathrm{~dB}$ with 8.6 cm-long Er/Yb doped glass planar amplifier," in OFC Tech. Dig., 1998, pp. $45-48$.

[2] W. J. Miniscalco, "Erbium-doped glasses for fiber amplifiers at 1500 nm," J. Lightwave Tech., vol. 9, pp. 234-250, Feb. 1991.
[3] K. Hattori, T. Kitagawa, M. Oguma, Y. Ohmori, and M. Horiguchi, "Erbium-doped silica-based waveguide amplifier integrated with a 980/1530 nm WDM coupler," Electron. Lett., vol. 30, pp. 856-857, 1994.

[4] K. Shuto, K. Hattori, T. Kitagawa, Y. Ohmori, and M. Horiguchi, "Erbium-doped phosphosilicate glass wave-guide amplifier fabricated by PECVD," Electron. Lett., vol. 29, no. 2, pp. 139-141, 1993.

[5] Y. C. Yan, A. J. Faber, H. deWaal, P. G. Kik, and A. Polman, "Erbium-doped phosphate glass waveguide on silicon with $4.1 \mathrm{~dB} / \mathrm{cm}$ gain at $1.535 \mu \mathrm{m}$," Appl. Phys. Lett., vol. 71, pp. 2922-2924, 1997.

[6] R. N. Ghosh, J. Shmulovich, C. F. Kane, M. R. X. de Barros, G. Nykolak, A. J. Bruce, and P. C. Becker, "8-mV threshold Er/sup 3+/-doped planar waveguide amplifier," IEEE Photon. Technol. Lett., vol. 8, pp. 518-520, Apr. 1996.

[7] K. Hattori, T. Hittagawa, M. Oguma, H. Okazaki, and Y. Ohmori, "Optical amplification in $\mathrm{Er}^{3+}$-doped $\mathrm{P}_{2} \mathrm{O}_{5}-\mathrm{SiO}_{2}$ planar waveguides," $J$. Appl. Phys., vol. 80, no. 9, pp. 5301-5308, 1996.

[8] G. Nykolak, P. C. Becker, J. Shmulovich, Y. H. Wong, D. J. DiGiovanni, and A. J. Bruce, "Concentration-dependent ${ }^{4} \mathrm{I}_{13 / 2}$ lifetimes in $\mathrm{Er}^{3+}$-doped fibers and $\mathrm{Er}^{3+}$-doped planar waveguides," IEEE Photon. Technol. Lett., vol. 5, pp. 1014-1016, Sept. 1993.

[9] E. M. Yeatman, "Sol-gel fabrication for optical communication components: prospects and progress," in Proc. SPIE Crit. Rev. Proc. CR-68, S. I. Najafi and M. Andrews, Eds., 1997, pp. 119-142.

[10] A. Holmes, R. R. A. Syms, M. Li, and M. Green, "Fabrication of buriedchannel waveguides on silicon substrates using spin-on glass," Appl. Opt., vol. 32, no. 25, pp. 4916-4921, 1993.

[11] R. R. A. Syms, A. S. Holmes, W. Huang, V. M. Schneider, and M. Green, "Development of the SC-RTA process for fabrication of sol-gel based silica-on-silicon integrated optic components," J. Sol-Gel Sci. Technol., vol. 13, pp. 509-516, 1998.

[12] X. Orignac, D. Barbier, X. M. Du, and R. M. Almeida, "Fabrication and characterization of sol-gel planar waveguides doped with rare-earth ions," Appl. Phys. Lett., vol. 69, pp. 895-897, 1996.

[13] J. Xu, S. Aubonnet, H. F. Barry, and B. D. MacCraith, "Preparation and characterization of erbium-doped ormosil planar waveguides," Mat. Lett., vol. 57, pp. 4276-4281, 2003.

[14] G. A. Kumar, R. Riman, E. Snitzer, and J. Ballato, "Solution synthesis and spectroscopic characterization of high $\mathrm{Er}^{3+}$ content $\mathrm{LaF}_{3}$ for broadband $1.5 \mu \mathrm{m}$ amplification," J. Appl. Phys., vol. 95, pp. 40-47, 2004.

[15] P. Etienne, P. Coudray, J. Porque, and Y. Moreau, "Active erbium-doped organic-inorganic waveguide," Opt. Commun., vol. 57, pp. 4276-4281, 2003.

[16] E. M. Yeatman, M. M. Ahmad, O. McCarthy, A. Vannucci, P. Gastaldo, D. Barbier, D. Mongardien, and C. Moronvalle, "Optical gain in Er-doped $\mathrm{SiO}_{2}-\mathrm{TiO}_{2}$ waveguides fabricated by the sol-gel technique," Opt. Commun., vol. 164, pp. 19-25, 1999.

[17] W. Huang, R. R. A. Syms, E. M. Yeatman, M. M. Ahmad, T. V. Clap, and S. M. Ojha, "Fiber-device-fiber gain from a sol-gel erbium-doped waveguide amplifier," IEEE Photon. Technol. Lett., vol. 14, pp. 959-962, July 2002.

[18] F. d'Acapito, S. Mobilio, P. Gastaldo, D. Barbier, L. F. Santos, O. Martins, and R. M. Almeida, "Local order around Er3+ ions in $\mathrm{SiO}_{2}-$ $\mathrm{TiO}_{2}-\mathrm{Al}_{2} \mathrm{O}_{3}$ glassy films studied by EXAFS," J. Non-Cryst. Solids, vol. 293, pp. 118-124, 2001.

[19] M. A. Fardad, E. M. Yeatman, E. J. C. Dawnay, M.Mino Green, and F. Horowitz, "Effects of $\mathrm{H}_{2} \mathrm{O}$ on structure of acid catalyzed $\mathrm{SiO}_{2}$ sol-gel films," J. Non-Cryst. Solids, vol. 183, pp. 260-267, 1995.

[20] K.Kazuo Arai, H.Hiroshi Namikawa, K.Ken Kumata, and T.Tatsutoku Honda, "Aluminum or phosphorus co-doping effects on the fluorescence and structural properties of neodymium-doped silica glass," J. Appl. Phys, vol. 59, no. 10, pp. 3430-3436, May 1986.

[21] J. Laegsgaard, "Dissolution of rare-earth clusters in $\mathrm{SiO}_{2}$ by Al codoping: a microscopic model," Phys. Rev. B, vol. 65, pp. 174 114-1-174 114-10, Apr. 2002.

[22] F. Di Pasquale and M. Zoboli, "Analysis of erbium-doped waveguide amplifiers by a full-vectorial finite-element method," J. Lightwave Technol., vol. 11, pp. 1565-1573, Oct. 1993.

[23] M. Federighi and F. Di Pasquale, "The effect of pair-induced energy transfer on the performance of silica waveguide amplifiers with high $\mathrm{Er}^{+3} / \mathrm{Yb}^{+3}$ concentrations," IEEE Photon. Technol. Lett., vol. 7, pp. 303-305, Mar. 1995.

[24] F. Di Pasquale, M. Zoboli, M. Federighi, and I. Massarek, "Finite-element modeling of silica waveguide amplifiers with high erbium concentration," IEEE J. Quantum Electron., vol. 30, pp. 1277-1282, May 1994. 
[25] C. Strohhofer and A. Polman, "Relationship between concentration in $\mathrm{Er}^{3+}-\mathrm{Yb}^{3+}$ doped waveguide amplifiers," J. Appl. Phys., vol. 90, no. 9, pp. 4314-4320, Aug. 2001.

[26] C. E. Chryssou, F. Di Pasquale, and C. W. Pitt, "Improved gain performance in $\mathrm{Yb}^{3+}$ sensitized $\mathrm{Er}^{3+}$-doped alumina $\left(\mathrm{Al}_{2} \mathrm{O}_{3}\right)$ channel optical waveguide amplifiers," J. Lightwave Technol., vol. 19, pp. 345-349, Oct. 2000.

[27] W. Huang and R. R. Syms, "Analysis of folded erbium-doped planar waveguide amplifiers by the method of lines," J. Lighwave Technol., vol. 17, pp. 2658-2664, Dec. 1999.

[28] J. Nilsson, B. Jaskorzynska, and P. Blixt, "Performance reduction and design modification of erbium-doped fiber amplifiers resulting from pair-induced quenching," IEEE Photon. Technol. Lett., vol. 5, pp. 1427-1429, Dec. 1993.

[29] M. J. F. Digonnet, M. K. Davis, and R. H. Pantell, "Rate equations for clusters in rare earth-doped fibers," Opt. Fiber Technol., vol. 1, pp. 48-58, August 1994.

[30] M. K. Davis, M. J. F. Digonnet, and R. H. Pantell, "Characterization of clusters in rare earth-doped fibers by transmission measurements," $J$. Lightwave Technol., vol. 13, pp. 120-126, Feb. 1995.

[31] F. Di Pasquale and M. Federighi, "Modeling of uniform and pair-induced upconversion mechanisms in high concentration erbium-doped silica waveguides," J. Lighwave Technol., vol. 13, pp. 1858-1864, Sept. 1995.

[32] J. L. Wagener, P. F. Wysocki, M. J. F. Digonnet, and H. J. Shawn, "Modeling of ion pairs in erbium-doped fiber amplifiers," Opt. Lett., vol. 19, no. 5, pp. 347-350, Mar. 1994.

[33] M. P. Hehlen, N. J. Cockroft, T. R. Gosnell, A. J. Bruce, G. Nykolak, and J. Shmulovitch, "Uniform upconversion in high concentration $\mathrm{Er}^{3+}$ doped soda lime silicate and aluminosilicate glasses," Opt. Lett., vol. 22, no. 11, pp. 772-775, June 1997.

[34] M. W. Sckerl, S. Guldberg-Kjaer, M. R.M. Rysholt Poulsen, P. Shi, and J. Chevallier, "Precipitate coarsening and self organization in erbiumdoped silica," Phys. Rev. B, vol. 59, pp. 13 494-13 497, June 1999.

[35] V. A. Borodin, K. H. Heinig, and S. Reiss, "Self-organization kinetics in the finite precipitate ensembles during coarsening," Phys. Rev. B, vol. 56, pp. 5332-5344, Sept. 1997.

[36] J. Philipsen, P. Gastaldo, C. Cassagnettes, D. Barbier, A. Kevorkian, and A. Yeniay, "Self-referenced Q-switched pump-probe transmission experiment for the determination of the degree of clustering in Er-doped planar waveguides," in Proc. OSA Trends in Optics and Photonics, Optical Amplifiers and Their Applications, , vol. 30, 1999, pp. 80-83.

[37] T. Ohtsuki, S. Honkanen, S. I. Najafi, and N. Peyghambarian, "Cooperative upconversion effects on the performance of $\mathrm{Er}^{3+}$ doped phosphate glass waveguide amplifiers," J. Opt. Soc. Amer. B, vol. 14, pp. 1838-1845, July 1997.

[38] S. Taccheo, G. Sorbello, S. Longhi, and P. Laporta, "Measurement of the energy transfer and upconversion constants in Er-Yb doped phosphate glass," Opt. Quantum Electron., vol. 31, pp. 249-262, Oct. 1998.

[39] Y. Yan, A. J. Faber, and H. de Waal, "Luminescence quenching by $\mathrm{OH}$ groups in highly Er-doped phosphate glasses," J. Non-Cryst. Solids, vol. 181, pp. 283-290, 1995.

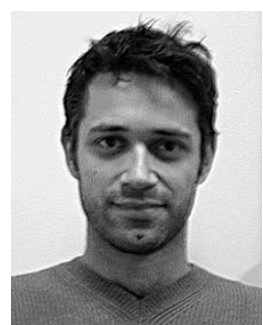

Athanasios Laliotis was born in Athens, Greece, in 1975. He received the B.Sc. degree in physics from the University of Athens, Athens, Greece, in 1998 and the M.S.E. degree in electrical engineering from Princeton University, Princeton, NJ, in 2000. He is currently working toward the Ph.D. degree at Imperial College London, London, U.K.

He was with Demokritos Research Centre, Athens, Greece, as a Research Assistant between 2000 and 2001. His scientific interests lie in the field of optical communications and integrated optics, with emphasis on planar waveguide amplifiers.

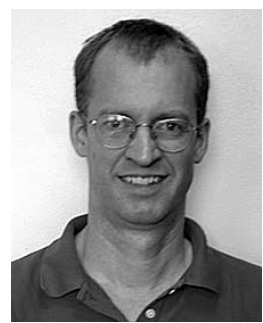

Eric M. Yeatman received the B.Eng. degree in engineering physics and the M.Sc. degree in physics from Dalhousie University, Halifax, NS, Canada, in 1985 and 1986, respectively, and the Ph.D. degree from Imperial College London, London, U.K., in 1989.

Since then, he has been a Member of Staff with the Electrical and Electronic Engineering Department, Optical and Semiconductor Devices Group, Imperial College London, currently as a Reader in Microengineering and Deputy Head of Group. His current research includes micromechanical actuators and generators, microstructures for optical and microwave applications, and integrated optical amplifiers.

Munir M. Ahmad received the Ph.D. degree in chemistry from Durham University, U.K. His dissertation focused on the synthesis and electrical and magnetic properties of organic semiconductors.

He has worked on the design, synthesis, and application of electro-active and specialty polymers. He joined the Optical \& Semiconductor Devices Group, Department of Electronic and Electrical Engineering, Imperial College London, London, U.K., in 1993 and has worked on a number of projects related to microengineering, microelectromechanical systems and materials for optical devices.

Dr. Ahmad is a Chartered Chemist and a member of the Royal Society of Chemistry.

Weibin Huang was born in Yantai city, China. He received the B.Eng. and M.Sc. degrees from Tsinghua University, Beijing, China, in 1984 and 1986, respectively, and the Ph.D. degree from Imperial College London, London, U.K., in 1999.

From 1986 to 1994 , he worked as a Lecturer with the Department of Electronic Engineering, Tsinghua University. From 1999 to 2001, he was with the Department of Electronics and Electrical Engineering, Glasgow University, Glasgow, U.K., before returning to Imperial College, where he is currently a Research Associate. His research interest is in the field of integrated optics and micromachining. 